\title{
Investigations with All Optical Binary Subtractor Network at 100Gbps
}

\author{
Devendra Kr. Tripathi*
}

Dept. of Electronics \& Communication, S.I.E.T Allahabad, U.P (India) 211012(U.P)

Received: 05 May 2017; Accepted: 06 August 2017; Published: 08 November 2017

\begin{abstract}
This article demonstrates for the proposed all optical binary subtractor network. Performance explored by exploiting nonlinear attribute the cross gain modulation with semiconductor optical amplifier which otherwise considered as cons if used as an amplifying tool. Numerical simulations have been executed, accordingly accustomed data inputs at $100 \mathrm{Gbps}$ data rate employed, generated aspired difference and the borrow outputs concurrently. The design's performance for the non return to zero, return to zero and Manchester modulation formats have been analyzed, according to it is key performance parameter the extinction ratio. Investigations inferred optimum performance for the non return to zero modulation format outcome extinction ratio of $13.5 \mathrm{~dB}$, together with difference and borrow output have been successfully confirmed. Further designs execution with non return to zero modulation format for the key parameters range of data rates, laser peak power, the normalizer power, pump current, the current injection efficiency and the modulator bias voltage have been evaluated. Evaluation inferred that optimum performance is subject to the pertinent election of the vital design parameters. The proposed design is simpler by virtue of key attributes low power consumption and stability altogether with higher integration capability as well provides output generation simultaneously at higher data rate. It was lacking with preceding elucidations. Accordingly, this illustration could be an assistance towards novel contemporary complex digital optical computing combinational networks.
\end{abstract}

Index Terms: Optical Binary Subtractor (OBS), Semiconductor optical amplifier (SOA), All-Optical Logic (AOL), Mach-Zehnder Interferometer (MZI), cross gain modulation (XGM).

(C) 2017 Published by MECS Publisher. Selection and/or peer review under responsibility of the Research Association of Modern Education and Computer Science

\section{Introduction}

Accompanied by evolution of contemporary technologies over the past few decades extensive operations of point-to-point multiplexing technologies for the data transportation have been observed. Inherited by the * Corresponding author:

E-mail address: 
excellent attributes of fiber domain numerous fiber optical communication schemes exercised with their relevant pros and cons. Though, with hasty growth of subscribers, internet based applications have further heightened need for the speed and spectrum [1].However, preexisting core network's electronic devices as switches and routers are not amply swift and inept to endure with any supplementary hike in speed along with inefficient optoelectronic translation. As, it requires transforming optical (O) signal to electronic (E) signal and then back to optical signal. So, endeavors on to work out for all-optical logic (AOL) systems are mounting briskly, which can inherit full benefit of optical domain.Accordingly,elaboration of photonics signal at the optical layer is striking for performing diverse commutating executions and surmount all the limitations regarding speed of electronics. Altogether with growth in optical amplifier technology there is huge swing towards design of all optical computing circuits with semiconductor optical amplifier (SOA) owing to its fast nonlinenear action and reduction in optoelectronic transformation immensely, well put into designs to accomplish the commendable operations. Studied effect of changing duty cycle on BER performance of FSO link with hybrid amplifier for varied attenuation successfully. Designs studied with SOA as active nonlinear elements together with Mach-Zehnder interferometer (MZI) met well for the all optical gate operations. MachZehnder Interferometer (2x2) practiced to design as optical switch, model with faster switching capability and reliability. All optical switching action explored with SOA and optical fiber for non return to zero pattern at 20Gbps data rate resulted with good performance [2-5]. As well performance was evaluated with optical fiber and wavelength translation method relying upon the semiconductor optical amplifiers [6-10]. By using SOA Mach-Zehnder interferometer configuration for all optical inverter at low data rate have resulted in good optical performance metric[11]. Whereas all-optical buffer and OR logic gates has been realized using Mach-Zehnder interferometer design at $10 \mathrm{Gbps}$, explored extinction ratio performance of 19.21 and $17.61 \mathrm{~dB}$ [12].In another approach studied for the optical gates utilizing quantum-dot semiconductor optical amplifiers (QD-SOAs) as nonlinear elements in Mach-Zehnder Interferometer (MZI).The optical gates were designed and evaluated for the Boolean operations, logic gate, employed as core logic units to structure the complex circuits[13]. While the design for all optical NOR gate with Fabry-Perot (FP) laser chip with two MEMS tunable lasers and compared with other fiber-based gating mechanism. Experimentally evaluated performance at 100Mbps showed more than $20 \mathrm{~dB}$ extinction ratio performance [14].Accordingly, numerous optical gates were exercised, the essential units to execute combinational circuits. For that all optical half adder with polarization-shift-keying (PolSK) modulation format and semiconductor optical amplifiers was realized[15].Further, numerous approaches were exercised for the half adder by means of ultrafast nonlinear interferometers (UNI), using terahertz optical asymmetric demultiplexer (TOAD) and using a PPLN waveguide outcome with good performance[16-19]. Another combinational design for all-optical half-adder, subtractor, OR logic gate at 40Gbps investigated altogether with one periodically poled lithium niobate (PPLN) waveguide. Exploiting SFG and DFG method create the Carry and Borrow outputs showed noteworthy performance[20].Further, optical combinational circuit realization with one PPLN, one SOA design other with one PPLN, and two SOA designs employing waveguide resulted in acceptable half-subtractor, half-adder operations and decoder showed for 10Gbps [21-22]. Now a days globally there is brisk transformation from analog to digital technology for that researchers are incessantly working for the analog to digital transformation schemes. Accordingly, digital wallet has been demonstrated.Further, analog and digital OFDM schemes were demonstrated efficiently, with no frequency shifting occured in every sub-carrier and BER reduced in digital OFDM system over the analog OFDM system [23-24].Accordingly, with digital domain amongst many constuients of any digital design combinational circuits play a vital role, for that numerous investigations with combinational circuits have been carried out. However, in spite of tremendous investigation in the past there were certain limitations with preceding explorations. So, there is always a wide scope and aspire to find and project the unrevealed part in front of the briskly changing digital world. So, as to attain high data rate, increased reliability, cost reduction and optimal performance. As the semiconductor optical amplifier is an adaptable element which could be employed for a range of optical signal processing applications owing to its least space necessity, monolithic integration potential. 
So, in this prospect a combinational design for the optical domain is proposed with semiconductor optical amplifier and optical coupler for the optical binary subtractor (OBS) operation at higher data rate. For that performance is to be explored with significant design parameters. Accordingly, the paper is bifurcated in segments, as first section elucidates the introduction, section two for the schematics presentation, section three illustrate for the results and discussion and subsequently the conclusion part.

\section{The Design Presentation}

A combinational design proposed for the high speed all optical subtractor operation is revealed in the Figure1.It is based on the semiconductor optical amplifier, generates sum and carry output altogether in the two branches of the design. For the simulation of data input pulse pattern are generated by the pseudo random binary signal (PRBS) with defined pre and post bits, at different data rates for instance at 100Gbps and

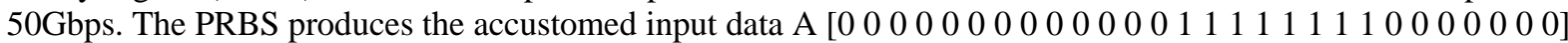
and data B [0 00000000001111000001111000000000$]$. In order to get good accuracy pre and post bits are inserted in the input data patterns.Generated data inputs are passed through the signal generator which generate the non return to zero, return to zero, Manchester modulation pattern and converts it to their corresponding pattern, driven by the raised cosine driver. This output signal is allowed to pass through Mach Zehnder modulator with bias voltage of 0.094 volts and 0.5 chirp factor. This signal is combined with continuous wave laser with relative intensity noise (RIN) of $-150 \mathrm{~dB} / \mathrm{HZ}$, generates laser pulse with peak power of 3 watt at the wavelength of $1550 \mathrm{~nm}$.It is referred to as probe signal. The combined laser and input modulated data is joined to a $2 \times 2$ coupler, which also couple the other continuous wave laser pulse with peak power of $0.1 \mathrm{e}-8$ watts.It is referred to as pump signal, which generates control pulse of $1555 \mathrm{~nm}$ wavelength. The laser powers are high and low so as to excite nonlinear characteristics in the nonlinear medium (SOA). The SOA's with different pump current are used one in the first branch to realize the difference output other in second branch for the generation of the borrow output.

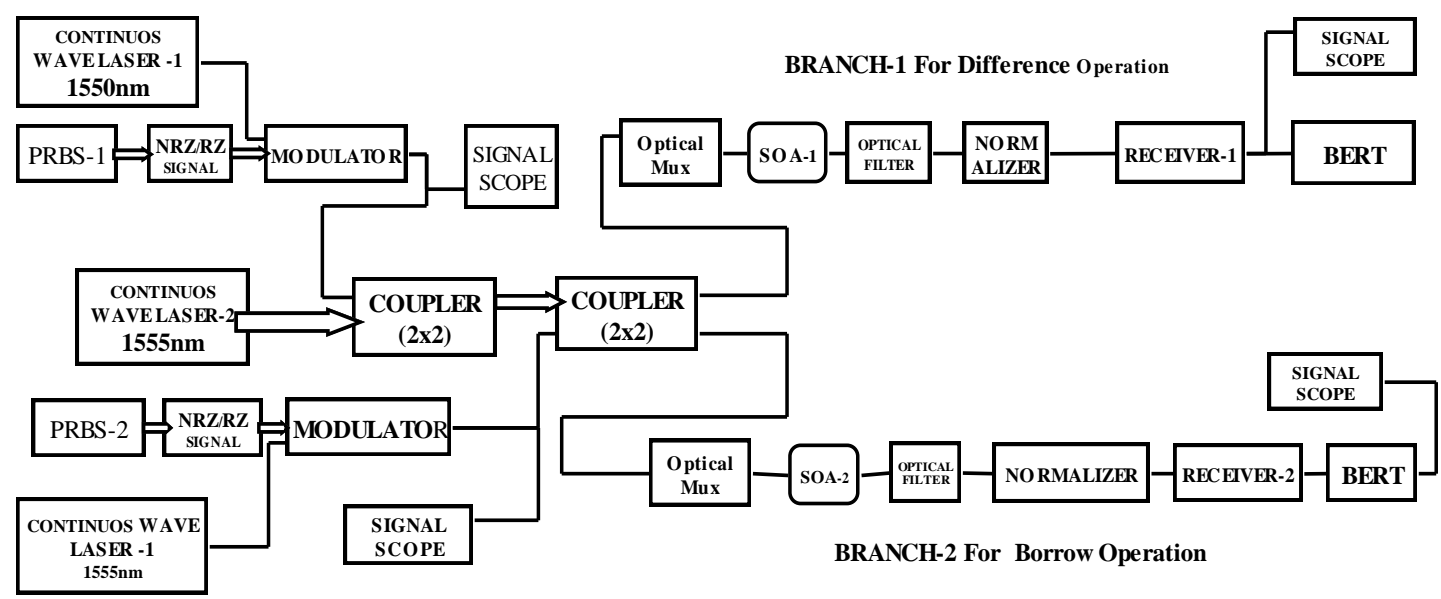

Fig.1. The optical subtractor schematic

Accordingly in the design the operating parameters of the first SOA were as the pump current of $1.35 \mathrm{~A}$, length as $5.0 \mathrm{e}-4 \mathrm{~m}$, thickness $1.5 \mathrm{e}-7 \mathrm{~m}$, width as $3.0 \mathrm{e}-6 \mathrm{~m}$, unity confine factor with line width enhance factor as 6 were picked. While the parameters for the other SOA in branch two was taken as the pump current of 0.09A, 
confine factor as 0.85 and other parameters are same as previously defined. The second modulated input data and the output of the first coupler $(2 \times 2)$ are allowed to pass through the next coupler $(2 \times 2)$. The output of this coupler is allowed to pass through first branch of the design with optical multiplexer and to the semiconductor optical amplifier. After propagating through SOA, light pulses go through the cross gain modulation (XGM) operation accountable for the needed optical operations. The two continuous wave laser signals referred as probe carry the data inputs A and Data B at the wavelength $1550 \mathrm{~nm}$ for the subtraction operation. Another laser source used is referred as the pump signal $(1555 \mathrm{~nm})$ with different power level than probe laser source. Now owing to higher laser power and the injected pump current in semiconductor optical amplifier its nonlinear attribute is incited. At the same time as the carrier dynamics within SOA is swift and gain counters in step with the variation of power input on a bit-by-bit basis. So there is depletion of carrier with altering the accessible carrier density for the amplification and accordingly diminishes the available gain. The gain diminution too relies upon the injection current applied to the active region of amplifier. Therefore powerful signal has an opposite effect on the gain obtainable to the frail signal. Consequently there is data transfer, generates optical subtractor logic action.

Accordingly, output of semiconductor optical amplifier in the branches is allowed to pass through the optical filter. For detection of numerous optical subtractor logics optical filter at right wavelength and bandwidth should be picked aptly, so as to realize the accurate results. It filters out $1555 \mathrm{~nm}$ wavelength resulting signal passed through the optical normalizer. Numerous design parameters are taken in order to optimize the performance. Ultimately to get the original signal it is allowed to propagate through the optical receiver which performs the detection operation for the required difference and borrow output signal. Finally, for the necessary performance measurement purpose the bit error tool is applied. To observe the output waveform pattern scope signal is used, which produce for the necessary output measured performance.

\section{Results and Discussion}

The combinational circuits are the vital element for modern digital processing systems. Accordingly for the digital optical computing system, amongst numerous basic arithmetic operations, subtraction is one of the key operations. The optical binary subtractor is one of the basic unit to form the complex computing building blocks, for that an optical subtractor diagram is illustrated in the Fig.1. In the design for the performance

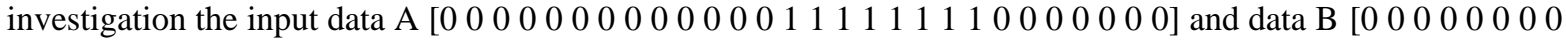
011111000001111100000000 ] were used and their corresponding outputs are as illustrated in the Table.1.At the 100Gbps data rate of operation numerically simulated resulting output waveform pattern, for the difference and borrow operations are demonstrated in the Fig.2 and Fig.3.As for the design of any optical link optical modulation format is one of the significant factor. So, for these data inputs, operation at 100Gbps links performance with non return to zero (NRZ), return to zero (RZ) and Manchester modulation formats is investigated. As the design is composed of numerous components and an appropriate selection of their parameters is a matter vital consideration. Intended, for the performance optimization as it makes a design noteworthy and well-organized. Accordingly, the optical subtractor performance is furthermore investigated at the $100 \mathrm{Gbps}$ and $50 \mathrm{Gbps}$ operating data rate with non return to zero modulation format in support of the numerous imperative design issues. For that numerous observations are taken and explored in the Fig.4 to Fig.14 in terms of extinction ratio(ER) parameter as shown in equation (1). Extinction ratio is elected as optimization norm for the best possible link's performance. E.R. must be as high as viable.It is expressed as ratio of minimum peak output power of "one" to extreme output peak power of "zero" in decibel(dB) revealed as,

$$
E R=\left(\frac{P_{\min }^{1}}{P_{\max }^{0}}\right) d B
$$


Table 1.

\begin{tabular}{|c|c|c|c|c|c|c|}
\hline S.n & Pre bits & & & & & Post bits \\
\hline $\begin{array}{c}\text { Data A } \\
\text { At 1550nm }\end{array}$ & $\mathbf{0 0 0 0 0}$ & $\mathbf{0 0 0 0}$ & $\mathbf{0 0 0 0}$ & $\mathbf{1 1 1 1}$ & $\mathbf{1 1 1 1}$ & $\mathbf{0 0 0 0 0 0 0}$ \\
\hline $\begin{array}{c}\text { Data B } \\
\text { 1550nm }\end{array}$ & $\mathbf{0 0 0 0 0}$ & $\mathbf{0 0 0 0}$ & $\mathbf{1 1 1 1}$ & $\mathbf{0 0 0 0}$ & $\mathbf{1 1 1 1}$ & $\mathbf{0 0 0 0 0 0 0}$ \\
\hline $\begin{array}{c}\text { Difference } \\
\text { output }\end{array}$ & $\mathbf{0 0 0 0 0}$ & $\mathbf{0 0 0 0}$ & $\mathbf{1 1 1 1}$ & $\mathbf{1 1 1 1}$ & $\mathbf{0 0 0 0}$ & $\mathbf{0 0 0 0 0 0 0}$ \\
\hline $\begin{array}{c}\text { Borrow } \\
\text { output }\end{array}$ & $\mathbf{0 0 0 0 0}$ & $\mathbf{0 0 0 0}$ & $\mathbf{1 1 1 1}$ & $\mathbf{0 0 0 0}$ & $\mathbf{0 0 0 0}$ & $\mathbf{0 0 0 0 0 0 0}$ \\
\hline
\end{tabular}

Data A

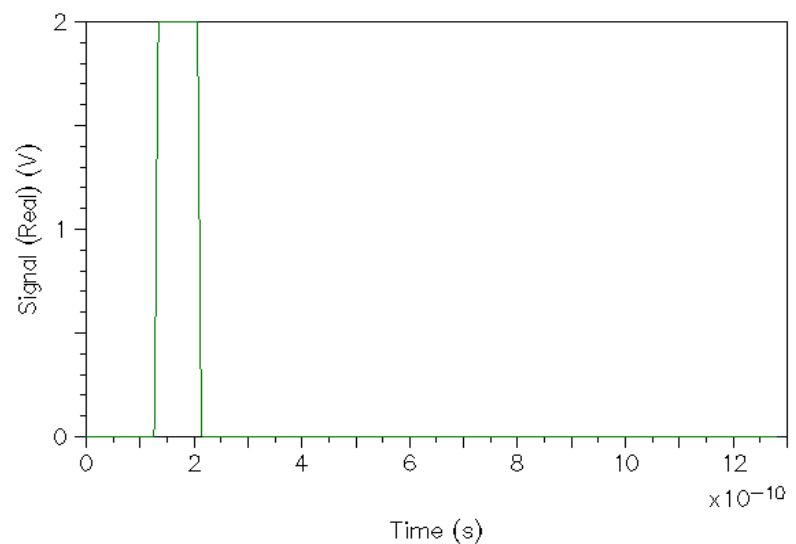

Fig.2. Data input A

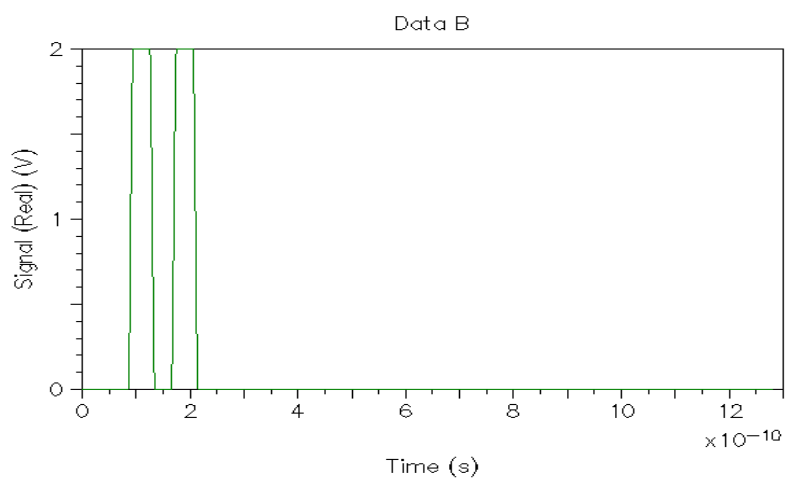

Fig.3. Data input B 


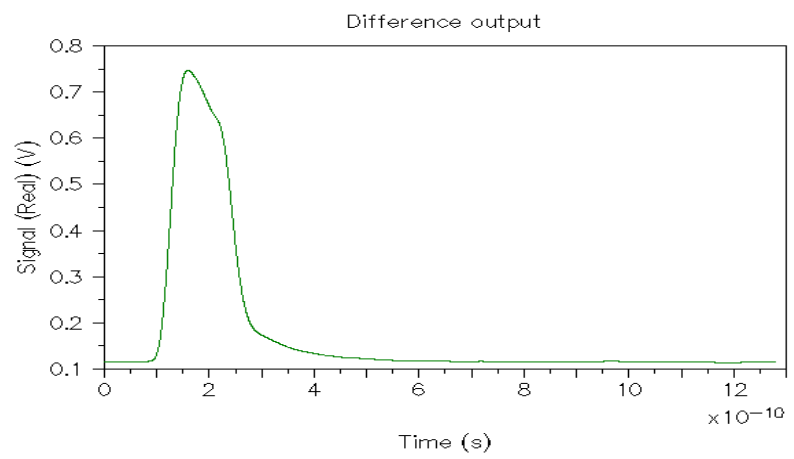

Fig.4. Difference output

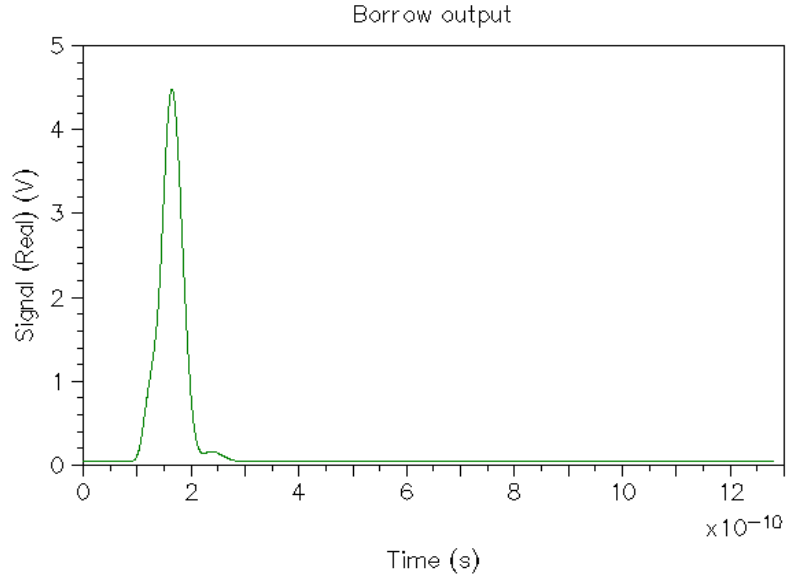

Fig.5. Borrow output

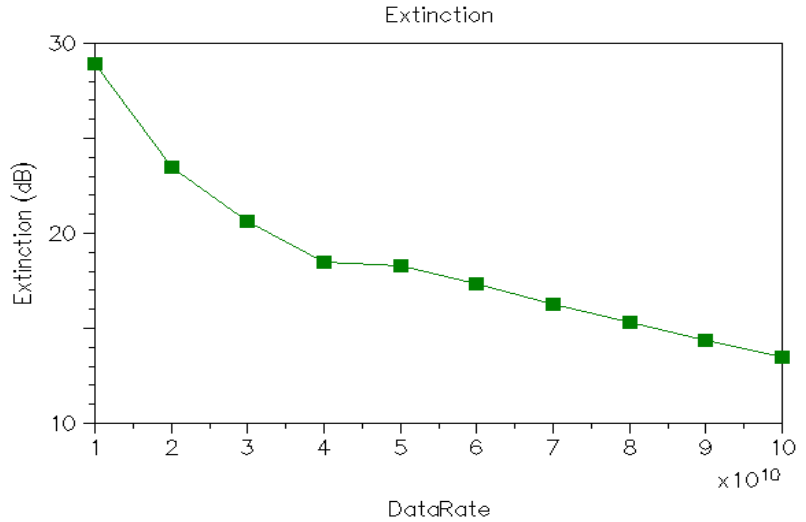

Fig.6. Extinction vs. Data rate for NRZ modulation 


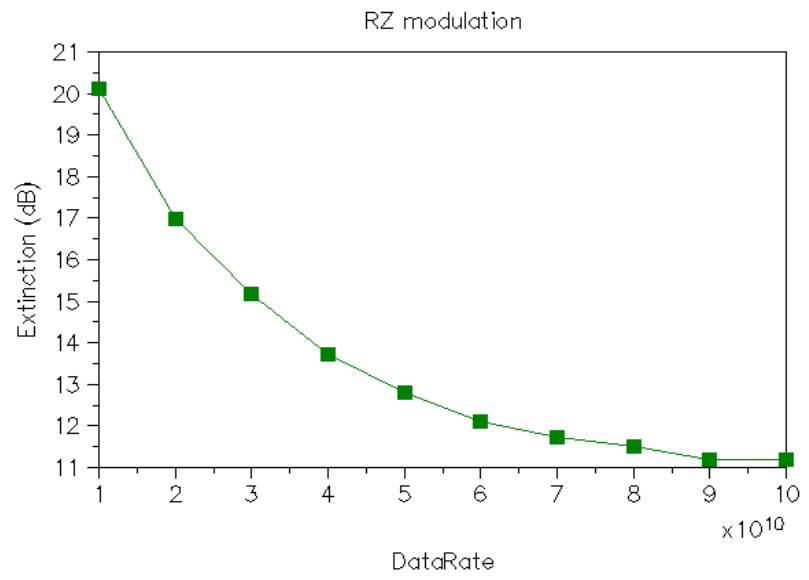

Fig.7. Extinction vs. data rate for RZ modulation

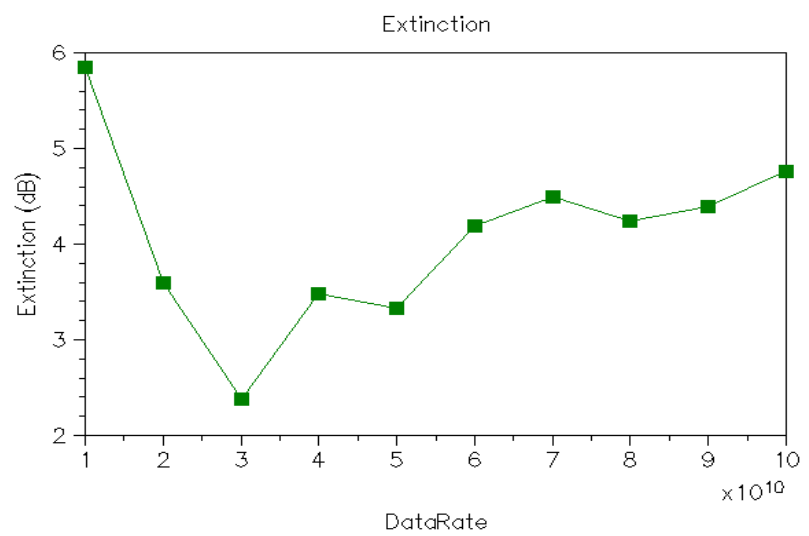

Fig.8. Extinction vs. data rate for Manchester modulation

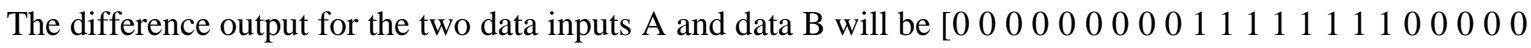
$\left.\begin{array}{llllll}0 & 0 & 0 & 0 & 0 & 0\end{array}\right]$, for that the corresponding output waveform pattern is shown in the Fig4. The borrow output for the given inputs will be [0 00000000011111000000000000000000 , for that corresponding waveform is displayed in the Fig.5.The optical performance parameter the extinction ratio is one of the significant specified parameter for the optical systems. Generally, digital optical processing links send binary data with two levels of optical power with high power level stands for a binary 1 and the low power level for a binary 0. Thus, the ratio between the "one" level and the "zero" level is referred to as extinction ratio. Accordingly, the simulated performance illustrated with Fig.6, Fig.7 and Fig.8. It illustrate performance of the optical subtractor link with non return to zero, return to zero and Manchester modulation formats for the variation of data rates. Results depict that performance in the case of Manchester modulation format is degraded. Whereas it illustrates nice extinction ratio (more than $10 \mathrm{~dB}$ ) performance for the non return to zero and return to zero modulation formats with range of data rates(10 to $100 \mathrm{Gbps})$. 


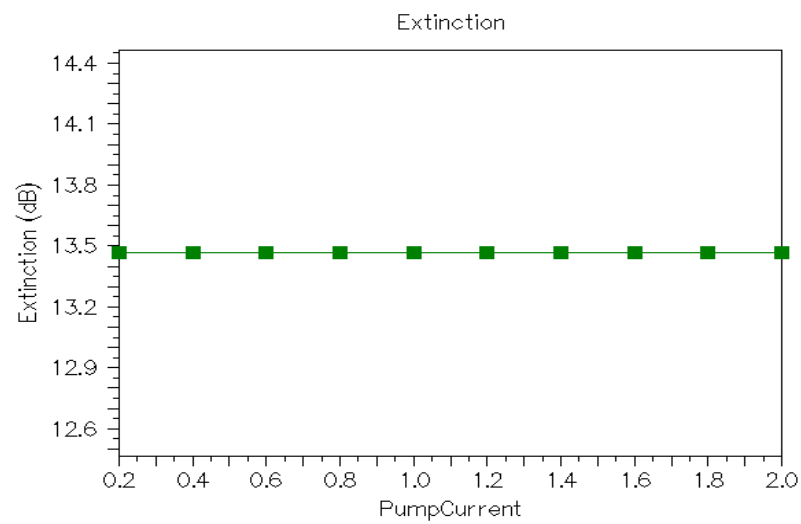

Fig.9. Extinction vs. pump current for NRZ modulation(100Gbps)

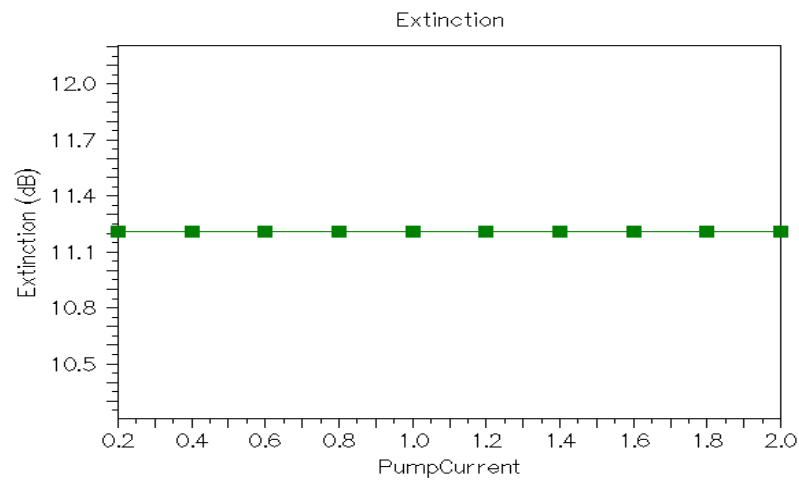

Fig.10. Extinction vs. pump current for RZ modulation

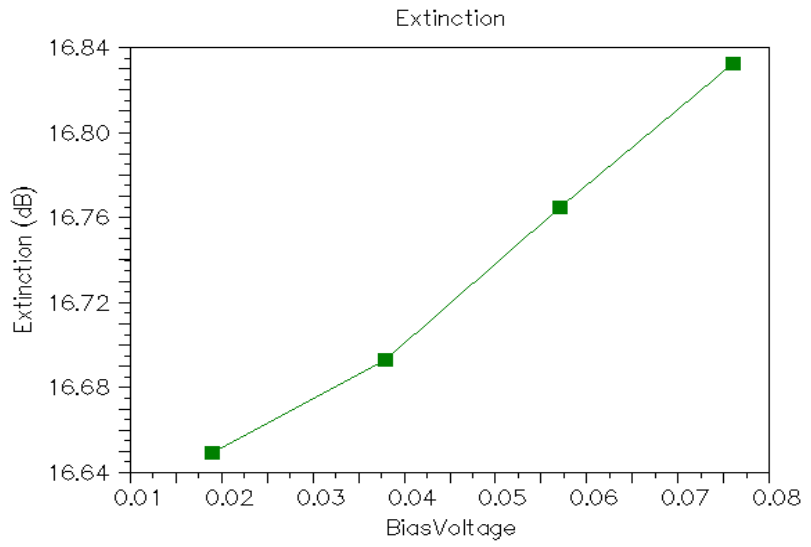

Fig.11. Extinction vs. Bias voltage (Modulator) for NRZ modulation (100Gbps) 


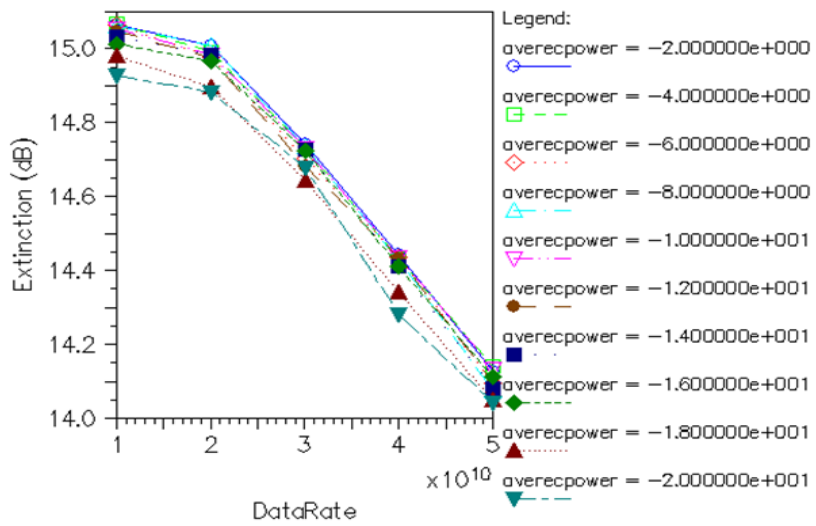

Fig.12. Extinction vs. Data Rate vs. normalizer power (NRZ modulation)

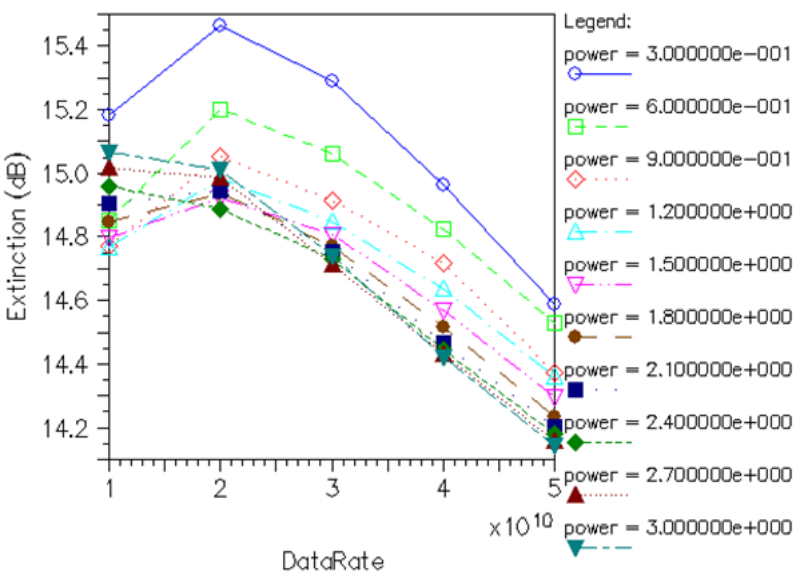

Fig.13. Extinction vs. Data Rate vs. laser power (NRZ modulation)

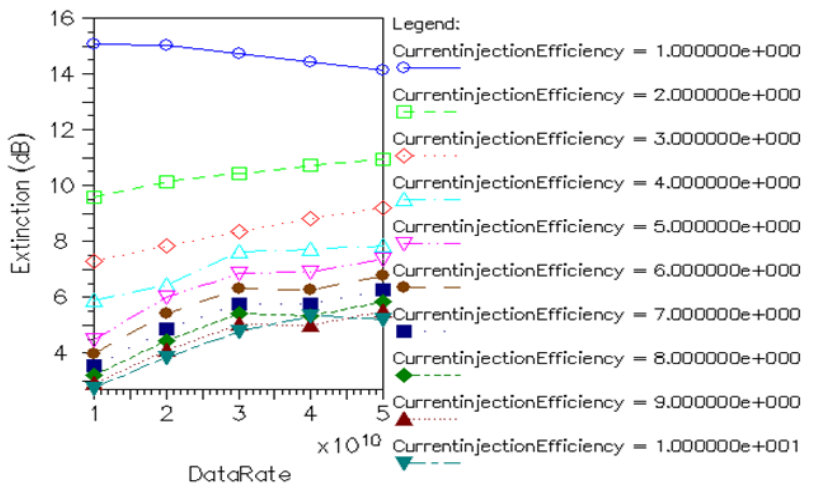

Fig.14. Extinction vs. Data Rate vs. current injection efficiency (NRZ modulation) 
However, for the semiconductor optical amplifier amongst numerous operating parameters pump current is one of the fundamental factors, which is accountable for the numerous optical operations. As for the population inversion the injected current must be ample and probability of stimulated emission is enormous in contrast to stimulated absorption, so it demonstrates optical gain. Accordingly numerical investigations for the NRZ and RZ modulation format at $100 \mathrm{Gbps}$ data rate with variation of the pump current of the semiconductor optical amplifier is demonstrated in the Fig.9 and Fig.10. Results illustrate good optical performance metric for the NRZ and RZ modulation formats. It depicts higher extinction (13.5dB) for the NRZ and 11.2dB for the RZ modulation format. It illustrtaes steady extinction ratio as there is depletion of the carrier concentration in the SOA active region which rely on pump current(SOA).Subsequently leading towards the saturation region.Another key optical component used with design is optical modulator the Mach Zehnder modulator, amongst various design issues selection of the modulator bias voltage is also a crucial factor. It is used to modulate the input data altogether with its choosen design factors. Accordingly links performance is explored forthe data rates and bias voltage. So for that investigated performance is illustrated in the Fig11.It depicts evaluated performance for the extinction factor against the bias voltage of the modulator at 100Gbps data rate employing the non return to zero modulation format. The result depicts good performance in terms of the excellent extinction ratio $(>10 \mathrm{~dB})$ performance. The signal normalizer is one of the vital constituent for the signal processing for that Fig.12 shows the performance investigation of optical subtractor with NRZ modulation format for the normalizer average power vs. data rate vs. the optical performance parameter the extinction ratio. It demonstrated good quality extinction ratio performance for the variation over range of data rates. As well it inferred that for good performance the normalizer average power is one of the important parameter and should be with appropriately selected.

A further significant performance measurement parameter for the optical system is the observation for the peak laser power. Accordingly investigation for NRZ modulation pattern for the peak power of the continuous wave laser connected with probe pulse is executed, as illustrated in Fig.13 for the data rate variation from 10 to 50Gbps. It inferred that for the low laser power value the optical subtractor's performance is good, showing high extinction factor.Accordingly optical semiconductor optical amplifiers current injection factor is one the essential consideration for the stimulation, owing to the injection of current into the active region of SOA. For that, the extinction performance were plotted as against the data rate as shown in the Fig.14.As the results showed strong variation in the extinction performance and for the value one of the current injection efficiency, showed very good output performance. The realization of optical binary subtractor has explored the likelihoods for increased complexity combinational logic operation and more options for manifold logic integration, that could promote for the other digital signal processing functions.

\section{Conclusions}

The all optical combinational high speed optical binary subtractor has been designed and its performance successfully investigated for the numerous crucial design parameters. The investigated performance for the optical subtractor with variation of data rate for the NRZ, RZ and Manchester modulation formats have been compared. It illustrated tremendous performance for the NRZ modulation pattern with optimum extinction ratio of $13.5 \mathrm{~dB}$ at $100 \mathrm{Gbps}$ data rate. Accordingly the sum and carry outputs for the optical subtractor with NRZ modulation is generated simultaneously and for that the resultant output waveform pattern has been confirmed. Investigations have been also carried out at $50 \mathrm{Gbps}$ data rate, demonstrated excellent performance metric with evaluated design factors. It has also inferred that proposed scheme with semiconductor optical amplifier, is one of the competent schemes with good potential for the ultrafast all-optical signal processing applications. 


\section{Acknowledgment}

Thanks to Department of Electronics and Communication University of Allahabad-India for providing the software OptSim(R-Soft) Fiber Optic Communication System.

\section{Ethical statement}

For the proposed work I have not taken any financial assistance from any agency/organization and conflict of interest as "NONE".

\section{References}

[1] D K Tripathi, et al. Study in F.O.C. Multiplexing Techniques-A Review. Journal of Electrical Engineering \& Electronic Technology 2014; 3(1):1-23.

[2] Dimitriadou E, Zoiros K E. On the feasibility of $320 \mathrm{~Gb} / \mathrm{s}$ all-optical and gate using quantum-dot semiconductor optical amplifier-based Mach-Zehnder interferometer. Progress in Electromagnetic ResearchB 2013; 50:113-140.

[3] Mørk J, Nielsen M L, Berg T W. The dynamics of semiconductor optical amplifiers: Modelling and applications. Optic express Photon 2003; 14(1): 42-48.

[4] D K Tripathi. Design and Investigations for SOA with Fibre as optical Switch. International Journal of Computer Science \& Engineering Technology (IJCSET) March 2016; 7(3):94-98.

[5] Pooja Kumari, Rajeev Thakur, BER Evaluation of FSO Link with Hybrid Amplifier for Different Duty Cycles of RZ Pulse in Different Conditions of Rainfall. I.J. Wireless and Microwave Technologies 2017; 1: $1-12$

[6] Kang B K, Kim J H, Byun Y T. All optical AND gate using probe and pump signals as the multiple binary points in cross phase modulation. Japanese Journal of Applied Physics 2002; 41(2):568 - 570.

[7] Lee S, Park J, Lee K., All optical exclusive NOR logic gate using Mach-Zender interferometer. Japanese Journal of Applied Physics 2002; 41(2):1155-1157.

[8] Lee H, Yoon H, Kim Y, Jeong J. Theoretical study of frequency chirping and extinction ratio of wavelength converted optical signals by XGM and XPM using SOA. IEEE Japanese Quantum Electron Aug1999; 35 (8):1213-1219.

[9] Durhuus T, Mikkelsen B, Joergensen C. All optical wavelength conversion by SOA. Journal of Light wave Technology1996; 14 (6):942-954.

[10] Stubkjaer K E. Semiconductor optical amplifier-based all optical gates for high-speed optical processing. IEEE Journal of Selected Topics in Quantum Electrons 2000; 6 (6): 1428-1435.

[11] P Singh, et al. Design and analysis of all-optical inverter using SOA-based Mach-Zehnder interferometer. Optik 2013, 124, (14):1926- 1929.

[12] Pallavi Singh, et al. Designs of all-optical buffer and OR gate using SOA-MZI. Optical \& Quantum Electronics 2014; 46 (11):1435-1444.

[13] Kyriakos E,Zoiros Z. All-Optical Logic Gates with Quantum-Dot Semiconductor Optical Amplifiers. ICTON- Greece 2013; 1-4.

[14] Liu B, Cai H, Zhang X M J. MEMS OPTICAL LOGIC NOR GATE USING INTEGRATED TUNABLE LASERS. in Proceedings of the IEEE $22^{\text {nd }}$ International Conf. on Micro Electro Mechanical Systems (MEMS '09),Sorrento, Italy2009;971-974. 
[15] Pei-li L, De-xiu H. Ultrahigh-speed all-optical half adder based on four-wave mixing in semiconductor optical amplifier. OSA2006; 14(24):11839-11847.

[16] A. J. Poustie, K. J. Blow, A. E. Kelly, et.al. All-optical binary half-adder.Opt.Communication 1998; 156:22-26.

[17] S. Kumar, D. Gurkan, A. E. Willner. All-optical half adder using a PPLN waveguide and an SOA, OFC 2004; 23-27.

[18] J. H. Kim, Y. T. Byun, Y. M. Jhon, et.al. All-optical half adder using semiconductor optical amplifier based devices, Opt. Communication 2003; 218: 345-349.

[19] Jian Wang, Junqiang Sun, Qizhen Sun, Single-PPLN-based simultaneous half-adder, half-subtracter and OR logic gate proposal and Simulation, Optical Society of America2007; 15(4):1690-9.

[20] S. Kumar, D. Gurkan, A. E. Willner, K. Parameswaran, M. Fejer, All-optical half adder using a PPLN waveguide and an SOA, OFC February2004;1:23-27.

[21] J. E. McGeehan, S. Kumar, A. E. Willner, All-optical digital half-subtracter/adder using semiconductor optical amplifiers and a PPLN waveguide, CLEO 2005, May2005; 2:1061-1063.

[22] Pallavi Singh et.al. Design and analysis of all-optical half-adder, halfsubtractor and 4-bit decoder based on SOA-MZI configuration. Optical Quant Electronics 2016; 48:159.

[23] Harshal R. Kanhekar, Sayali N. Mane.Digital Wallet. I.J. Wireless and Microwave Technologies 2015; 4: 62-68.

[24] Pravat Kanti Nath, M. M. Rahmanb et. Al. Performance, Analysis between Analog OFDM \& Digital OFDM. I.J. Wireless and Microwave Technologies 2016; 4: 84-100.

\section{Authors' Profiles}

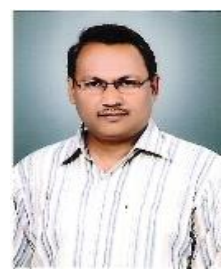

Devendra kr. Tripathi received his B.Sc, B.Tech, M. Tech and D.Phil degree in Electronics and Telecommunication engineering from the Department of Electronic \& Communication, University of Allahabad. He is presently faculty in electronics engineering department of S.I.E.T. Allahabad(U.P)India. His area of interest includes Wireless Communication Technology, Microwave and Fiber Optics Communication. He is life member of ISTE.

How to cite this paper: Devendra Kr. Tripathi," Investigations with All Optical Binary Subtractor Network at 100Gbps", International Journal of Wireless and Microwave Technologies(IJWMT), Vol.7, No.6, pp. 21-32, 2017.DOI: 10.5815/ijwmt.2017.06.03 